Prepared for the U.S. Department of Energy

under Contract DE-AC05-76RL01830

\title{
Design and Construction of a Gas Jet Target for RIB Experiments
}
U Greife
F Montes
KA Chipps
J Blackmon
M Smith
M Wiescher
DW Bardayan
M Couder
SD Pain
$\mathrm{P}$ Vetter
K Schmitt
A Lemut
H Schatz
LE Erikson

February 2011

\section{Pacific Northwest}

NATIONAL LABORATORY

Proudly Operated by Battelle Since 1965 


\title{
DISCLAIMER
}

This report was prepared as an account of work sponsored by an agency of the United States Government. Neither the United States Government nor any agency thereof, nor Battelle Memorial Institute, nor any of their employees, makes any warranty, express or implied, or assumes any legal liability or responsibility for the accuracy, completeness, or usefulness of any information, apparatus, product, or process disclosed, or represents that its use would not infringe privately owned rights. Reference herein to any specific commercial product, process, or service by trade name, trademark, manufacturer, or otherwise does not necessarily constitute or imply its endorsement, recommendation, or favoring by the United States Government or any agency thereof, or Battelle Memorial Institute. The views and opinions of authors expressed herein do not necessarily state or reflect those of the United States Government or any agency thereof.

\author{
PACIFIC NORTHWEST NATIONAL LABORATORY \\ operated by \\ BATTELLE \\ for the \\ UNITED STATES DEPARTMENT OF ENERGY \\ under Contract DE-AC05-76RL01830
}

Printed in the United States of America
Available to DOE and DOE contractors from the Office of Scientific and Technical Information,
P.O. Box 62, Oak Ridge, TN 37831-0062;
ph: (865) 576-8401
fax: $(865)$ 576-5728
email: reports@adonis.osti.gov

\begin{abstract}
Available to the public from the National Technical Information Service, U.S. Department of Commerce, 5285 Port Royal Rd., Springfield, VA 22161 ph: (800) 553-6847 fax: $(703) 605-6900$ email: orders@ntis.fedworld.gov online ordering: http://www.ntis.gov/ordering.htm
\end{abstract}

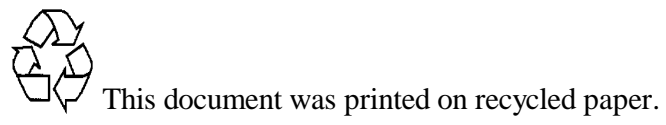




\section{Design and Construction of a Gas Jet Target for RIB Experiments}

$\begin{array}{ll}\text { U Greife } & \text { F Montes } \\ \text { KA Chipps } & \text { J Blackmon } \\ \text { M Smith } & \text { M Wiescher } \\ \text { DW Bardayan } & \text { M Couder } \\ \text { SD Pain } & \text { P Vetter } \\ \text { K Schmitt } & \text { A Lemut } \\ \text { H Schatz } & \text { LE Erikson }\end{array}$

February 2011

Prepared for the U.S. Department of Energy under Contract DE-AC05-76RL01830

Pacific Northwest National Laboratory

Richland, Washington 99352 


\title{
Design and Construction of a Gas Jet Target for RIB Experiments
}

\author{
Jet Experiments in Nuclear Structure and Astrophysics (JENSA) Collaboration \\ U. Greife ${ }^{1}$, K.A. Chipps ${ }^{1}$, U. Hager ${ }^{1}$, M.S. Smith², D.W. Bardayan², S.D. Pain², K. Schmitt² H. Schatz ${ }^{3}$, \\ F. Montes ${ }^{3}$, J.C. Blackmon ${ }^{4}$, M. Wiescher ${ }^{5}$, M. Couder ${ }^{5}$, P.A. Vetter ${ }^{6}$, A. Lemut ${ }^{6}$, L. Erikson ${ }^{7}$ \\ ${ }^{1}$ Colorado School of Mines ${ }^{2}$ Oak Ridge National Laboratory ${ }^{3}$ National Superconducting Cyclotron Laboratory \\ ${ }^{4}$ Louisiana State University ${ }^{5}$ University of Notre Dame ${ }^{6}$ Lawrence Berkeley National Laboratory ${ }^{7}$ Pacific \\ Northwest National Laboratory
}

\section{Introduction}

Scattering and transfer reaction measurements using both stable and radioactive ion beams require targets that are well confined, having an optimum balance of target nucleus number density and thickness to maximize count rates but minimize reaction product energy loss and straggling. In inverse kinematics this is difficult to achieve, since the light target isotopes necessary (hydrogen, helium) cannot be easily made into targets. Traditional solid targets are often plagued with contaminants (such as carbon and oxygen), or require backing materials like aluminum or nickel which contribute substantially to straggling and background. Gas targets, such as are necessary with helium, can eliminate some of the difficulties, but introduce others: a gas cell requires thin windows which worsen energy and angular resolution, and an windowless gas target (achieved via differential pumping) is too extended along the beam access to allow angular distributions to be measured.

An advantageous solution to these difficulties is the construction of a supersonic gas jet target, which allows for a high density of target nuclei within a highly confined region. No windows or backing materials are present to produce unwanted background, gas purity is high and the amount of contamination is well controlled, and the small target size allows for high resolution measurements of energy and angle. Laval nozzles provide the high density and small dimensions necessary for the jet target, and various pumping stages, in conjunction with a diaphragm compressor, handle the flow and recirculate the gas within the system. Targets of this type are well documented in the literature, using a wide variety of gases and target densities.

The use of a gas jet target with radioactive ion beams, which informs nuclear structure, nuclear reaction and nuclear astrophysics studies, presents different constraints than with previous experiments. Light target gases, with areal densities up to $1 \times 10^{19}$ nuclei $/ \mathrm{cm}^{2}$, are required for use with low-intensity radioactive ion beams. Also necessary is the use of large area, highly segmented silicon detector arrays, high-efficiency gamma arrays and novel heavy ion detectors to efficiently measure the reaction products. No gas jet to date has been designed which fulfills all of these requirements. The Gas Jet Target Working Group, led by researchers at the Colorado School of Mines, are optimizing just such a jet target for construction and use at both new and existing radioactive ion beam facilities.

\section{General Design Considerations}

There are four main considerations when designing, constructing and testing the gas jet: nozzle design, effective pumping outside the jet, accommodation of detector arrays and recoil detectors, and an effective recirculation and gas cleaning system. The basic design of the gas jet target involves a Laval nozzle with two receiver stages. The receivers are backed by a series of high-throughput roots blowers and dry screw pumps, which feed into a $\sim 50 \mathrm{~m}^{3} / \mathrm{hr}$ diaphragm compressor. The compressor provides the reservoir pressure necessary at the top of the jet. Gas chillers minimize straggling due to thermal energy in the gas. A rough schematic is displayed in Figure 1.

The remainder of the gas throughput, roughly $1 \%$, is pumped by a large turbomolecular pump directly on the target chamber, as well as a series of upstream and downstream pumping stages separated by constrictive apertures. Design of the apertures and pumping stages determines the pressure gradient along the beamline, as well as providing the high vacuum $\left(\sim 10^{-6}\right.$ Torr) needed to connect to the accelerator. Various pressure and flow diagnostics will be located as necessary throughout the system for monitoring and feedback control, as well as safety considerations.

Additionally, the design of the jet components and target chamber will be such that large silicon detector arrays, other charged particle arrays, and gamma-ray arrays can be accommodated and used. Different reaction 
studies will require slightly different arrangements of detector systems, but a general multi-array design will allow for further optimization as specific needs arise.

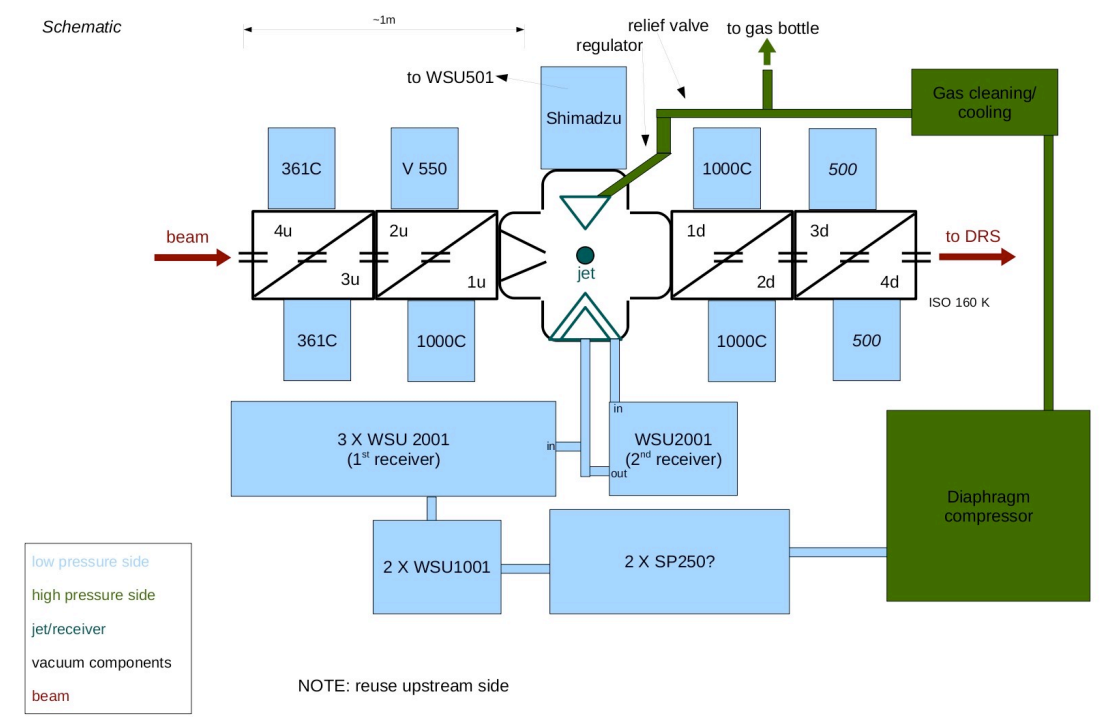

Illustration 1: Preliminary schematic of the gas flow components

\section{Optimization and Flexibility}

Due to the various types of reaction studies which would benefit from the use of a gas jet target, several are likely to be constructed over the coming years. The first will be installed at the target position of the Daresbury Recoil Separator (DRS) at the Holifield Radioactive Ion Beam Facility (HRIBF) at ORNL. The design requires that the downstream apertures of the pumping stages be wide enough to admit any recoils which fall inside the angular acceptance of the DRS. This affects the pumping required on the downstream side of the jet. It is envisioned that this target may be moved to TRIUMF in Vancouver, Canada, for use with the new EMMA recoil separator being designed and built in the ISAC-2 hall.

A second jet is being considered for the ReA3 facility at the NSCL. The preliminary implementation of this target would not require downstream pumping stages, because a beam diagnostic or ionization chamber would be located just downstream of the target chamber. The downstream portion of the ReA3 gas jet target will be designed in conjunction with the finalization of designs for the Separator for Capture Reactions (SECAR), the new recoil separator for FRIB, such that the target-detector system was optimized.

A third gas jet target may be built for the Dakota Ion Accelerators for Nuclear Astrophysics (DIANA) at the underground laboratory DUSEL in the Homestake Mine in South Dakota, in order to perform ultra-lowbackground reaction measurements. The DIANA facility is being designed to provide intense stable beam currents up to $100 \mathrm{~mA}$, and so a high-velocity gas jet target is absolutely necessary to handle the anticipated beam powers.

In each case, a balance must be found between the amount of flexibility desired (number of target gases the system is capable of handling, changes to pumping stages or apertures, changes to nozzle and thus jet dimensions, etc) and the amount of optimization desired (maximize density for one gas, design of apertures to complement recoil detectors, beam optics and infrastructure considerations, etc). This work is ongoing within the collaboration.

\section{Detector Systems}

It is envisioned that several detection systems will be either developed, adjusted or employed with the gas jet. Existing detector arrays, such as ORRUBA, SIDAR or CLARION, could be modified to fit around the jet for reaction studies, as described below. Arrays which are in the early stages, such as superORRUBA or SHARC, could also be modified for use with the jet, and designing mounts and feedthroughs for such arrays is planned. ANASEN or other heavy ion detectors could be placed downstream of the gas jet in place of pumping 
stages, and the jet could be used with or without a recoil separator.

Large arrays of charged-particle silicon-strip detectors will be implemented near the interaction point of the exotic beam with the gas jet target. These arrays are critical for determining the energy and angular distribution of ejectiles from the reactions of interest. The detection of the reaction products is required to extract the nuclear properties that determine the rates of reactions in astrophysical explosions.

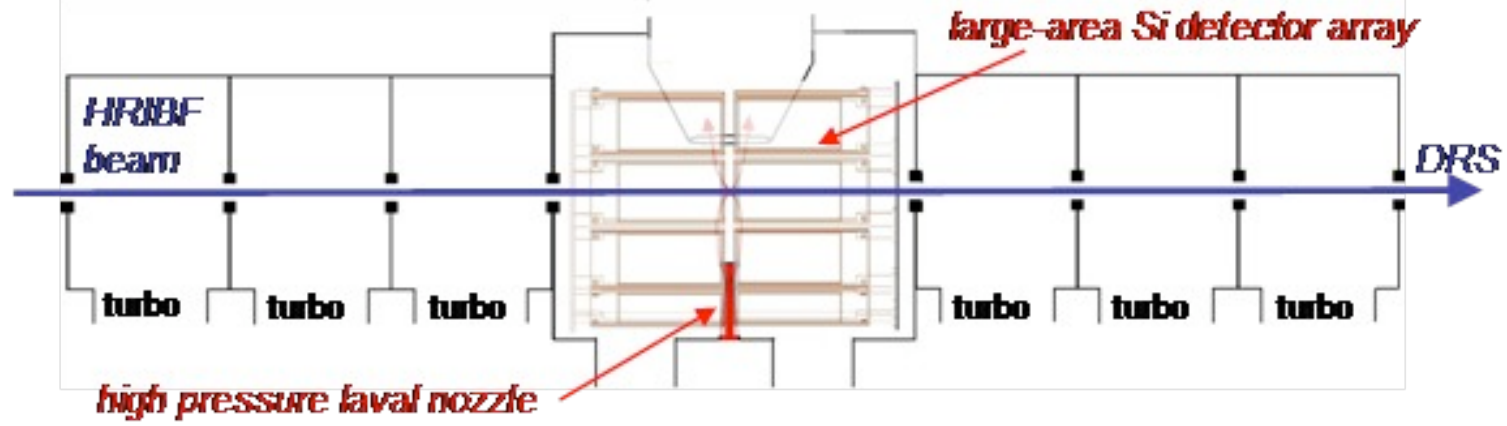

Illustration 2: The ORRUBA array coupled to the gas jet target

Large solid angle coverage is required to make most efficient use of the exotic beams available at HRIBF and to fully characterize the reactions under study. Therefore the existing arrays SIDAR and ORRUBA will be configured to surround the gas jet target. New mounting and cabling will be required in order accommodate the nozzle and gas receivers of the gas jet target. A possible design showing location of the ORRUBA detector in the gas system is shown in Fig. 2. SIDAR would be added in the chamber as end cap detectors. Additional detectors and signal processing electronics will be procured so that a full implementation of the SIDAR and ORRUBA arrays can be instrumented. Once in place, the detector arrays will be tested with the gas jet target and stable beams from the HRIBF in order to optimize solid angle coverage, resolution, and performance for the reactions of interest.

As ion beams have become more exotic, the beam intensities and contamination have made the ability to detect a gamma coincidence at the target into a requirement for many experiments. Additionally, the DRAGON separator at TRIUMF and the DRS at HRIBF have demonstrated that gamma arrays at the target are not only useful for event tagging, but the spectroscopy information can be used to produce meaningful science directly (for example by measuring short lived excited states).

Clearly, in the case of the gas jet target, a gamma spectroscopy array will be needed. We suggest the construction of a high efficiency and cost-efficient, close packed, HPGe array would best fill this need. One promising approach would be to enclose several germanium detectors within 2 custom cryostats to flank the gas jet. This gives great freedom in terms of custom geometry, crystal size, and crystal quality while maximizing detection efficiency by co-locating the crystals within distances of approximately $1 \mathrm{~cm}$ and utilizing no internal shielding. One example is the MARS array produced by Pacific Northwest National Laboratory (see Figure 3).

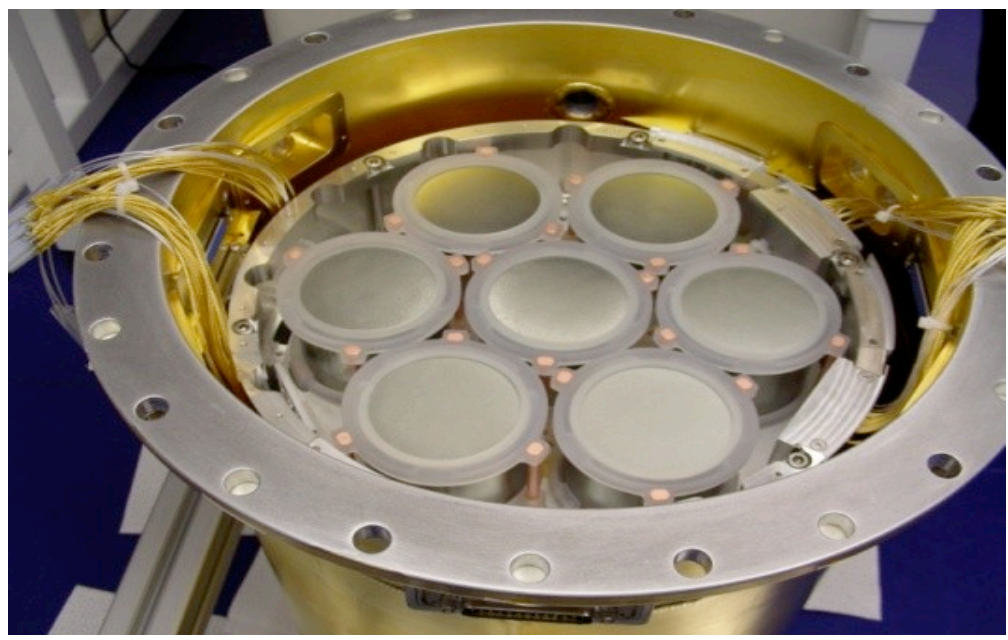

Illustration 3: The PNNL MARS array

At this time, members of the JENSA collaboration are looking to secure funding to run proof of concept 
measurements using the MARS array. This includes operating MARS at suitable beamline facilities such as ISAC1 at TRIUMF and also static laboratory tests with sources. Based on these results, a new array could be constructed at PNNL based on the same principles as the MARS array, but customized to the needs of the gas jet target. Funding for the design and procurement of the superORRUBA array exists, but ongoing funds for future operation or modification will be necessary. Implementation of these detection systems with the high-density gas jet target will create an opportunity for nuclear reaction studies unique in the world.

\section{Next Steps}

With the acquisition of components, construction and testing of the first gas jet target in the next year or two at ORNL, a wealth of useful information will be gathered to help in the final designs of the eventual FRIB jet. Gas flow calculations have been completed, quotes for the necessary components are currently being acquired, and infrastructure requirements are being finalized for the first (ORNL) jet. Members of JENSA will continue to work collaboratively on experimental campaigns, procurement of target gases, detector systems and specific design issues as they arise. A collaboration meeting is tentatively scheduled for March 25 ${ }^{\text {th }}, 2011$.

\section{Timelines, Budgets, Requirements}

The JENSA collaboration anticipates acquisition, construction and testing of the first (ORNL) and possibly second (ReA3) gas jet target within the next three years. This timeline is contingent upon appropriate levels of financial, engineering and administrative support; while the DOE grant to the Colorado School of Mines includes the design, purchase, construction and testing of one jet, additional manpower and budget is needed to simultaneously implement a second or third jet at the NSCL or DUSEL. Specific infrastructure requirements, beam optics considerations, experimental apparatus, available components and operational parameters vary slightly from facility to facility. For example, the jet being constructed at ORNL can take advantage of already existing pumps and pumping stages from the previous Windowless Gas Target (WGT), but equivalent pumps for ReA3 or DIANA may need to be purchased, and pumping stages need to be machined. While this does not necessarily double or triple the total gas jet expenditures, alternative sources of funding for the additional gas jets needs to be secured.

\section{Rough Timeline}

Spring 2011: begin acquiring components for ORNL gas jet target

machining of interior jet components determination of infrastructure needs

Summer 2011: acquisition of components (plus individual component testing)

Late 2011: machining of downstream pumping stages

Early 2012: $\quad$ preliminary testing of gas jet central region $\left({ }^{4} \mathrm{He}\right)$ acquisition of components for additional gas jets (ReA3, DIANA?)

Late 2012: $\quad$ acquisition and testing of compressor and gas purification systems machining of pumping stages and jet components for additional gas jets

2013: $\quad$ final on-site construction of ORNL gas jet testing of full gas jet system in-beam testing incorporation and testing of detector systems construction of additional gas jets

Rough Budget

Total:

CSM+subcontractors \$1.025million (including contingency; see Gas Jet Project Management Plan for detailed information)

\section{External Requirements}

Engineering work to determine correct infrastructure requirements at ReA3/FRIB

Engineering work to determine feasibility of/requirements for moving gas jet between facilities 